\title{
"Heteroatom-tagged" quantification of proteins via ICP-MS
}

\author{
Alfredo Sanz-Medel ${ }^{1}$
}

Published online: 17 June 2016

(C) Springer-Verlag Berlin Heidelberg 2016

Chemical speciation and speciation analysis of trace metals, concepts introduced more than 30 years ago, have evolved rapidly from toxicity and environmental fields to bioscience and biomedicine.

Nature has learned to use metals, even at very low levels, for very important biological functions, many of which are accomplished in the end through appropriate proteins. Therefore, metallo-proteins and their functions in life have become a hot topic in the field of speciation of biologically important heteroatoms (metals and semi-metals) naturally associated to proteins. To tackle such studies, hyphenated separations with ICP-MS elemental detection (e.g., HPLC-ICPMS) are the tools-of-the-trade, providing new insights to understand the role of metals in biology and medicine [1]. Traditionally, however, sulphur and phosphorous, two nonmetallic, very relevant "heteroatoms" (that is, elements other than the frequently found $\mathrm{C}, \mathrm{H}, \mathrm{O}$, and $\mathrm{N}$ in biomolecules) have been elusive to sensitive ICP-MS determinations. Fortunately, the recent introduction in the market of the ICP$\mathrm{MS}(\mathrm{Q}, \mathrm{Q}, \mathrm{Q})$, which uses a triple quadrupole as mass analyser for the ICP ion source, has enabled $\mathrm{S}$ and $\mathrm{P}$ elemental analysis at very low limits of detection (one to two orders of magnitude better than common quadrupole instruments). This sensitive $\mathrm{P}$ and $\mathrm{S}$ detection could extend substantially the usefulness of ICP-MS in protein analysis.

In fact, heteroatom-tagged targeted proteomics via ICP-MS [2] is proving to be invaluable for the screening of biologically relevant heteroatoms in complex mixtures of proteins and for

Alfredo Sanz-Medel

asm@uniovi.es

1 Department of Physical and Analytical Chemistry, Faculty of Chemistry, University of Oviedo, C/ Julián Clavería 8, 33006 Oviedo, Spain "absolute" quantifications of a known panel of targeted proteins (e.g., panel of biomarkers). Quantitative basic parameter evaluation in biological sample treatments and work-flows, in the activities of enzymes, as well as element effects in living organisms after administration of drugs or element supplementation are fields that have steadily increased in the last $5 \mathrm{y}$.

No doubt, phosphorous, sulphur, selenium, and, to a lesser extent, iodine, are the most promising natural tags for direct protein "absolute" quantifications, provided that the retention time and their stoichiometry within the targeted protein is known. Worth mentioning these days is the possibility to achieve absolute quantification of the protein (via determination of the $\mathrm{S}$ in biomolecule) and, virtually in a simultaneous manner, the determination of its total phosphorylation (global phosphorylation degree) by measuring the $\mathrm{P} / \mathrm{S}$ intensity ratios in the desired protein. Both parameters, absolute protein content (via $S$ ) and global phosphorylation degree of such protein (via $\mathrm{P} / \mathrm{S}$ measurement), can be obtained with a modern ICP$\mathrm{MS}(\mathrm{Q}, \mathrm{Q}, \mathrm{Q})$ at very low level and without resorting to any specific standard of the sought biomolecule (just using generic $\mathrm{S}$ and $\mathrm{P}$ standard calibration of the ICP-MS).

Of course, hetero-atom-tagged analysis requires a heteroatom, but if it is not naturally present in the protein(s), the biomolecule can still be labeled chemically with bifunctional reagents containing the heteroatom (detected by ICP-MS) and a biomolecule recognition moiety (e.g., an antibody).

This is a very similar approach to labeling in molecular mass spectrometry (e.g., ESI-MS), where using enriched non-metal isotopes for relative quantifications are commonplace (e.g., ICAT or SILAC techniques). When using ICP-MS for detection, a previous selective flow separation (e.g., HPLC) or, alternatively, a bioassay, are needed for adequate recognition of the required biomolecule (e.g., antibody reagents bound to the heteroatom tag). This second alternative of using bifunctional element/isotope coded affinity tags is 
now booming in the field of targeted proteomics with ICPMS. Such a promising tool, combining ICP-MS detection with immunoassays recognition, seems to offer notable and distinct advantages versus natural tags. Among them, I would underline the following: (a) increases in selectivity because of the biorecognition reaction; (b) new ways to achieve strong enhancements of the ICP-MS sensitivity and, so, of protein detection limits; (c) multiplexing capability, as we may take advantage of the multi-elemental/multi-isotopic character of the ICP-MS (with an excellent elemental mass resolution to avoid spectral overlaps); (d) improved measurement robustness derived from the "hard" ion source used; (e) straightforward novel applications of the isotope dilution analysis (IDA) concept and techniques [3]. This last aspect is critical to achieve "absolute" quantification of targeted proteins [2] and is gaining momentum as it could provide important advances in bioscience and biomedicine. Thus, an appropriate and controlled heteroatom labeling of proteins, e.g., via lanthanide polymer chelating probes [4], offers methodical analytical advantages compared with natural tags. In this line, the so-called "metal-coded affinity tag" (MeCAT), as inspired in the more common ICAT concept, should be mentioned because it may facilitate the important steps of protein preconcentration before final MS detection.

In any case, in contrast to natural metal tags enabling metallomics direct studies with ICP-MS, the labeling approach cannot offer direct information of the measured heteroatoms (usually non-existent) in the protein. However, labeling has been instrumental for the development of a revolutionary alternative to flow cytometry, the so-called single cell "mass" cytometry, based on ICP-MS final detection and is invaluable to achieve indirect multiplexing (using surrogate probes for a lot of key biological parameters to be measured in a single cell) not only for many proteins but for RNAs as well. In this way, further understanding of multi-parametric biological functions by broadening the experimental toolbox for systems biology investigations is possible [4]. In this vein, a bright future awaits these days for ICP-MS labeling using similar bifunctional approaches but with metal NPs as labels instead of metal-chelating polymers, for innovative further amplifications of ICP-MS signals. Such achievable extreme sensitivity has proven to be decisive in determining early alarm biomarkers of diseases (e.g., cancer and ocular diseases). This promising approach is still in its infancy. For a decisive quantum leap in the practical exploitation of NPsbased bio-nanotools in bioscience and proteomics, further fundamental research on degrees of labeling, stoichiometry, probes stability, etc. are urgently needed.

Novel applications of labeling to targeted protein analysis are growing quickly and are not restricted to just heteroatomtagged determinations of protein amounts. Activity determinations of enzymes (e.g., proteases) or multiplex determinations of several DNA targets (via hybridization and IDA measurements) have appeared in recent literature. In fact, the present potential of ICP-MS has been recently demonstrated for accurate targeted cell quantifications [5] after appropriate biospecific interactions of the reagent probes. More and more complementary detectors are used to study single cells, not just to enable simultaneous analysis of more than 30 molecular parameters in a single cell [4], but also using trifunctional probes for revealing and counting cancer cells (from thousands down to a few dozens of them) by the use of ICP-MS [5]. In this sense, targeting especially relevant cells (e.g., circulating tumor cells) could be a future application of elemental MS, complementary to the more common ESI- or MALDI-MS molecular techniques.

In conclusion, literature of the last $5 \mathrm{y}$ demonstrates that ICP-MS, the elemental version of molecular MS, is able to simplify the complexity of natural protein or peptide mixtures and so help to overcome many difficult analytical hurdles derived from the complexity. New instrumental advances, sample treatment methods, analytical labeling approaches, bioinformatics, etc. are facilitating the eventual acceptance and natural incorporation of ICP-MS into the numerous analytical fields of modern protein analysis [6].

I do expect to witness in the near future many and varied applications of elemental MS, in particular, for "absolute" quantitative heteroatom-tagged targeted proteomics, a clearly expanding field where ICP-MS offers a tailored tool for innovative insights and applications (e.g., more sensitive, robust, specific, and reproducible determinations of disease biomarkers).

\section{References}

1. Sanz-Medel A. From metalloproteomics to heteroatom-tagged proteomics. Anal Bioanal Chem. 2005;381:1-2.

2. Sanz-Medel A. Heteroatom(isotope)-tagged proteomics via ICPMS: screening and quantification of proteins and their posttranslational modifications. Anal Bioanal Chem. 2008;391: 885-94.

3. Bettmer J. Application of isotope dilution ICP-MS techniques to quantitative proteomics. Anal Bioanal Chem. 2010;397:3495-502.

4. Bendall SC, Simonds EF, Qui P, Amir EAD, Krutzik PO, Finck R, et al. Single-cell mass cytometry of differential immune and drug responses across a human hematopoietic continuum. Science. 2011;332:687-96.

5. Yan X, Yang L, Wang Q. Detection and quantification of proteins and cells by use of elemental mass spectrometry: progress and challenges. Anal Bioanal Chem. 2013;405:5663-70.

6. Chahrour O, Cobice D, Mallone J. Stable isotope labeling methods in MS-based quantitative proteomics. J Pharm Biomed Anal. 2015;113: $2-20$. 


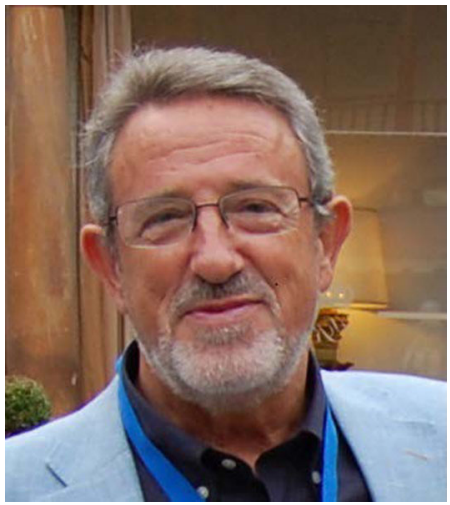

Alfredo Sanz-Medel has been Professor in the Department of Physical and Analytical Chemistry of Oviedo University, Spain, since 1982. After completing his Ph.D. in 1973, he carried out postdoctoral work on atomic fluorescence at Imperial College of Science and Technology in London. Back in Spain, he became Assistant Professor of Analytical Chemistry at Complutense University (Madrid) in 1975, and in 1978 he moved to Oviedo as an Associate Professor. His present research interests include the following main lines: 1. Development of new atomic detectors and analytical methodologies for ultratrace elemental analysis using spectrochemical plasmas (GD-OES, ICP-MS, GD-MS). 2. New molecular sensors, particularly those based on the use of quantum dots for bionano applications using molecular and elemental detection (luminescence and ICP-MS). 3. Hybrid techniques development, coupling a separation unit and an atomic detector, for ultratrace and trace metal analysis and their speciation to solve biological, biomedical, and environmental problems. 4. Speciation for proteomics, integrating MS "molecular" (MALDI-TOF and electrospray-Q-TOF) and "atomic" (ICP-MS) metal and semi-metal biomolecules, promoting the extensive use of "heteroatom-tagged proteomics" and bionano tagging approaches for early alarm diseases biomarkers. He is author and co-author of around 650 scientific publications in international journals, several patents, and a number of books. International prizes and awards received include the "Robert Kellner Lecture" (2007), European Winter Conference Award on Plasma Spectrochemistry (2011), Chemistry EuCheMS Award, Belgrade (2011), and Colloquium Spectroscopicum Internationale Award, CSI Award, Coimbra (2015). He has been one of the editors of Analytical and Bioanalytical Chemistry (ABC) since 2003. 